\title{
Coordination of eye and finger movements in copytyping
}

\author{
ALBRECHT WERNER INHOFF and TA-SING CHIU \\ State University of New York, Binghamton, New York \\ and \\ JIAN WANG \\ Hangzhou University, Hangzhou, People's Republic of China
}

\begin{abstract}
Eye movements and manual keypresses of one high-skilled typist were analyzed to determine eye-hand span, viewing time, and typing time for word and letter units. The results revealed a relatively small eye-hand span of 2.4 character spaces. Fixation time and typing time were positively correlated when word and letter units were analyzed. However, the correlation accounted for $79 \%$ of the variability $(r=.89)$ when word units were analyzed and for only $29 \%$ of the variability $(r=.54)$ when letter units were analyzed.
\end{abstract}

Champion typists are able to type up to 200 words per minute (wpm), which corresponds to about $60 \mathrm{msec}$ per keystroke. This high-speed motor behavior is impressive when the complexity of the skill is considered. During copytyping, typists have to encode the visually presented material via a sequence of eye movements, select specific sequences of manual keystrokes, direct the selected fingers to specific locations on a keyboard, and order the to-beexecuted keystrokes along a fine-grained temporal dimension.

Lashley (1951) was the first to argue that this high level of skill is possible only when the typist engages in some type of anticipatory motor planning. Rather than execute each keystroke as a separate functional unit, expert typists were assumed to prepare (order) sequences of keystrokes prior to response execution.

Examination of eye-movement records (Butsch, 1932) showed that the eyes of skilled typists are about 5 to 7 characters ahead of the currently executed keystroke, revealing some delay between the visual encoding of text and the corresponding keypress responses. Using a window technique, in which each manual keystroke advanced a window of legible text by one character (no text was available outside the window), Shaffer (1973) and Salthouse (1984) showed that highly skilled typists reach normal typing rates when the window of text is at least 8 characters ahead of the typed letter. Typing speed decreased when smaller window sizes were used. Eyemovement records and window studies thus converge in showing that high-speed typing is possible only when there is some delay between the visual encoding of to-be-typed

This research was supported by Grant HD24796 from the National Institute of Child Health and Human Development. Requests for reprints should be sent to Albrecht Inhoff, Department of Psychology, SUNYBinghamton, Binghamton, NY 13901. letters and words and the execution of the corresponding keypresses. This delay may be used to prepare (program) the sequence of to-be-executed keystrokes. The preparation of to-be-executed keystrokes could precede subsequent keypress execution by a relatively constant interval (and comprise a relatively constant number of intervening keystrokes), which represents Butsch's (1932) view, or it could comprise linguistic units of variable size, such as syllables, morphemes, or words.

If preparatory processes comprise a relatively constant number of keystrokes, then skilled typists should use a constant eye-hand span during the copytyping of text. If, however, linguistic units of variable size form the functional unit of response preparation, then a relatively variable eye-hand span should be obtained. Furthermore, if preparatory processes are applied to a relatively constant number of to-be-executed keystrokes (letter units), then viewing time per letter unit and subsequent typing time per letter unit should be highly correlated. If, however, linguistic units such as words form the functional unit of motor preparation, then viewing time and typing time should be highly correlated when word units are examined. The present experiment was designed to test these possibilities.

\section{METHOD}

\section{Subject}

One high-skilled typist, with a copytyping rate of approximately $90 \mathrm{wpm}$, participated in the study. A number of single-subject examinations of highly skilled typists have been reported in the literature.

\footnotetext{
Apparatus

Except for the first letter of each sentence, text was displayed in lower case. A 70-Hz VGA color monitor, controlled by a VGA graphics card $(640 \times 480$ pixel resolution), was used to display the to-be-typed text. Text was shown in light green on a black background. This display arrangement generated the same high-quality character display as is available with commercial word processing packages (in VGA monochrome display mode). The distance between the typist's eyes and the monitor
} 
was set at $65 \mathrm{~cm}$. At this viewing distance, each character subtended approximately $.33^{\circ}$ of visual angle.

Eye movements and eye fixations were recorded via a dual-Purkinje SRI eye-tracking system. The system, which has a spatial resolution of $10^{\prime}$ of arc, was used to sample eye position every millisecond. Manual keypresses were recorded on a Keytronics keyboard for the IBM AT. A Logitech mouse, which served as a supplementary buttonpush panel, was used to initiate and terminate the visual presentation of to-be-typed text.

For purposes of data reduction, eye-fixation durations and manual interkeypress times were measured to the nearest $.01 \mathrm{sec}$ and fixation positions were measured in 1-character units. A Dell 310 computer equipped with a 16-bit Data Translation A/D converter was used to link the monitor, the eye-tracking system, the mouse, and the keyboard.

\section{Materials and Procedure}

Twenty-five sentences, each containing from five to nine words, were used. All sentences were simple declarative sentences. None of the sentences was familiar to the subject.

A calibration of the eye-tracking system began the experiment. During the calibration, the subject was requested to fixate sequentially four briefly illuminated monitor positions (left, right, top, bottom), as they successively appeared on the screen. After calibration was completed, six character-size fields (top, bottom, left, right, 1 in. to the left of the screen center, and 1 in. to the right of the screen center) were illuminated in light green, and the subject was asked to fixate all six illuminated character positions in a prespecified order. During this calibrationcheck phase, the subject's eye position was plotted on the screen so that it appeared as a white cross that moved in synchrony with the eyes. The calibration check revealed a 0-letter horizontal/0-letter vertical error for four of the six illuminated character positions and a 1-letter horizontal/0-letter vertical error for two of the six fixation positions.

After calibration, the subject was asked to rest both hands on the keyboard, with the left and right index fingers on the " $f$ " and " $j$ " keys, respectively. The keyboard was placed on a small table, which was outside the subject's range of vision. After this, the experimenter depressed a mouse key to display a to-be-fixated marker on the left side of the screen. Upon the subject's fixation of the marker (as indicated by a fixation cross), the experimenter depressed another mouse key to display a to-be-typed sentence. Eye fixations were not visible during sentence typing. During copytyping, each keypress was echoed back to the screen, forming a line of letters $4 \mathrm{~cm}$ below the to-be-typed sentence. After sentence typing was completed, the experimenter used a mouse key to terminate the visual display. Another mouse-key response triggered the display of the left fixation marker. Again, a calibration check was performed, followed by the presentation of a to-be-typed sentence.

\section{RESULTS}

All beginning and ending words of each sentence and all incorrectly typed words were deleted from the main analyses. Furthermore, no eye-fixation times and no typing times were computed for word units that were not fixated during sentence typing. As a result, we obtained usable data from 80 words.

Eye-fixation times per fixated word were calculated. To account for differences in word length, a second measure was obtained, indexing fixation times per letter for each fixated word. Analogously, two estimates of word typing time were computed, comprising total typing time per word and interkeypress times per letter during word typing. The results showed a fixation time of $694 \mathrm{msec}$ per word and a fixation time of $133 \mathrm{msec}$ per character. The corresponding word and letter typing times amounted

\section{TYPING TIME AND EYE-HAND SPAN}

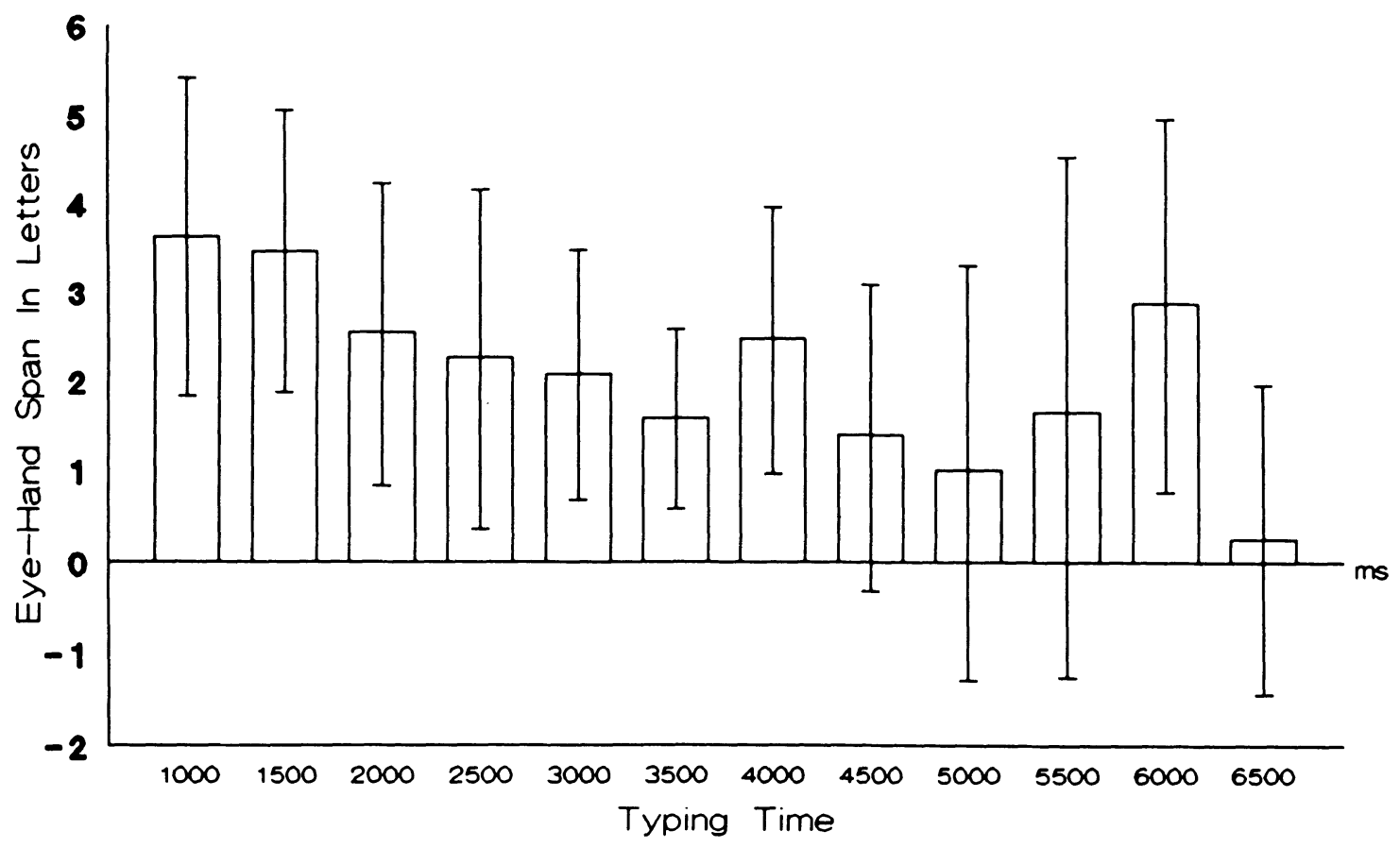

Figure 1. The eye-hand span as a function of typing time (in 500-msec units). 
to 631 and $118 \mathrm{msec}$, respectively. There was a strong positive correlation between word viewing time and word typing time $(r=.89, p<.001)$, accounting for $79 \%$ of the variability. The correlation was somewhat less, but still highly significant, when viewing and typing times per character were analyzed $(r=.54, p<.001)$, accounting for $27 \%$ of the variability.

A supplemental analysis was applied to the sentenceinitial fixation duration and the sentence-initial keypress. This analysis revealed considerably longer initial keypress times $(801 \mathrm{msec})$ than initial fixation times $(287 \mathrm{msec})$, presumably because the typist prepared a sequence of keypresses prior to typing onset.

The eye-hand span was measured every $500 \mathrm{msec}$, $1 \mathrm{sec}$ after the typing of the sentence had been initiated. Thus, every $500 \mathrm{msec}$, we determined the identity of the fixated letter and the identity of the concurrently typed letter. The distance between the two letter units (in terms of intervening letters) was used to index the eye-hand span. The lowest number of eye-hand span estimates per sentence was 9 , the highest number was 17 . The results are shown in Figure 1.

On the average, the eyes were only 2.4 character spaces ahead of the executing hand. Furthermore, there was a considerable amount of variability in the eye-hand span data. On some occasions, the eyes preceded the hand by as much as 7 character spaces; on other occasions, the hand slightly preceded the eyes.

\section{DISCUSSION}

Analyses of the sentence-initial fixation and keystroke indicated that considerably more time was needed to execute the first keypress than to execute the first saccade: The typist viewed a sequence of letters, and may have prepared (ordered) a sequence of to-be-executed keystrokes, prior to the execution of the first keystroke. Consistent with earlier results, the present eye-hand span data also show that the eyes remained ahead of manual keypresses throughout sentence typing.
The significant correlation between word viewing times and subsequent word typing times is in general agreement with the view that the oculomotor system and the manual motor system are coordinated during copytyping. Furthermore, the higher correlation between viewing time and typing time when word units were analyzed than when letter units were analyzed suggests that words may constitute the functional unit of keypress preparation. The assumption that words, rather than letters, constitute the basic unit of eye-hand coordination during copytyping may also account for the large variability in the eye-hand span. For instance, typists could attempt to keep the eyes approximately one word ahead of the currently typed word. The eye-hand span would then be relatively small when a short word is followed by another short word, but it would be relatively large when a long word is followed by another long word.

It should be noted that the present estimate of the eye-hand span is smaller than Butsch's (1932) original estimate. According to Butsch, high-speed typists (typing 70-100 wpm) should use an average eye-hand span of more than 6 character spaces. However, it appears possible that Butsch's method overestimated the eye-hand span. As indicated before, recent window studies (Shaffer, 1973; Salthouse, 1984) indicated that highly skilled typists asymptote normal typing rates when 8 or more character spaces are visible to the right of the currently typed letter (Shaffer, 1973; Salthouse, 1984). Thus, a preview of 8 characters represents the maximum range of useful visual information. If typists obtained more useful visual information in only a few instances, a larger window than 8 character spaces would have been needed for normal typing. Given that some useful visual information is obtained to the right of each fixation position, the eye-hand span of skilled typists should thus rarely exceed 5-7 character spaces, as was found in the present study.

\section{REFERENCES}

Bursch, R. L. C. (1932). Eye movements and the eye-hand span in typewriting. Journal of Education Psychology, 23, 104-121.

LASHLEY, K. S. (1951). The problem of serial order in behavior. In L. A. Jeffress (Ed.), Cerebral mechanisms in behavior (pp. 112-136). New York: Wiley.

SAlthouSE, T. A. (1984). Effects of age and skill in typing. Journal of Experimental Psychology: General, 113, 345-371.

SHAFFER, L. H. (1973). Latency mechanisms in transcription. In S. Kornblum (Ed.), Attention and performance (Vol. 4, pp. 435-447). New York: Academic Press.

(Manuscript received October 27, 1989.) 